\title{
Circular Holes Punched in a Magnetic Circuit used in Microspeakers to Reduce Flux Leakage
}

\author{
Dan-Ping Xu ${ }^{1}$, Yuan-Wu Jiang ${ }^{1}$, Han-Wen Lu ${ }^{1}$, Joong-Hak Kwon ${ }^{2}$, and Sang-Moon Hwang ${ }^{1 *}$ \\ ${ }^{1}$ Mechanical Engineering, Pusan National University, Busan 46241, Korea \\ ${ }^{2}$ Research and Development Center, EM-TECH, Changwon-si, Gyeongsangnam-do 51539, Korea
}

(Received 9 May 2016, Received in final form 7 September 2016, Accepted 7 September 2016)

\begin{abstract}
Lower flux leakage designs have become important in the development of microspeakers used in thin and miniaturized mobile phones. We propose four methods to reduce the flux leakage of the magnetic circuit in a microspeaker. Optimization was performed based on the proposed approach by using the response surface method. Electromagnetic analyses were conducted using the finite element method. Experimental results are in good agreement with the simulated results obtained in one degree-of-freedom analysis from 100 to $5 \mathrm{kHz}$. Both the simulated and experimental results confirm that one of the proposed methods is much more effective in reducing flux leakage than the other methods. In the optimized method, compared with a default approach, the average radial flux density in the air gap decreased only by $5.5 \%$, the maximum flux leakage was reduced by $28.6 \%$, and the acoustic performance at primary resonance decreased by $0.45 \mathrm{~dB}$, which gap is indiscernible to the human ear.
\end{abstract}

Keywords : microspeaker, reduce flux leakage, magnetic circuit, electromagnetic analysis, sound pressure level

\section{Introduction}

With the widespread use of electronic devices, thinner and smaller mobile phones offered competitive acoustic performance are in greater demand. A magnet with a larger volume, or a higher magnet grade, is usually used to improve the acoustic performance of a miniaturized microspeaker module [1-3], and results in a higher flux leakage as well. The arrangement of a microspeaker in a mobile phone is shown in Fig. 1(a). The sound holes are arrayed in front of a microspeaker unit, and the flux leakage measurement position is $1 \mathrm{~mm}$ over the speaker unit at the side of the sound holes. The flux leakage in a microspeaker is defined as the magnetic flux that does not pass through the air gap of the voice coil or through other parts of the magnetic circuit, as shown in Fig. 1(b). Dust can accumulate through the sound holes because of the flux leakage, especially the strongest flux leakage at the side of the sound holes. The accumulated dust harms the performance of a microspeaker, and can even jam the sound holes and damage the mobile phone. In this study,

CThe Korean Magnetics Society. All rights reserved.

*Corresponding author: Tel: +82-51-510-3204

Fax: +82-51-510-3204, e-mail: shwang@pusan.ac.kr a magnetic circuit with two $\mathrm{N} 48 \mathrm{H}$ grade magnets and a $41.5 \mathrm{~mm}^{3}$ volume of IM was set as the default design used in the microspeaker unit. The electromagnetic analysis is shown in Fig. 2 and the related parameters are shown in Table 1. On the basis of the default magnetic circuit,

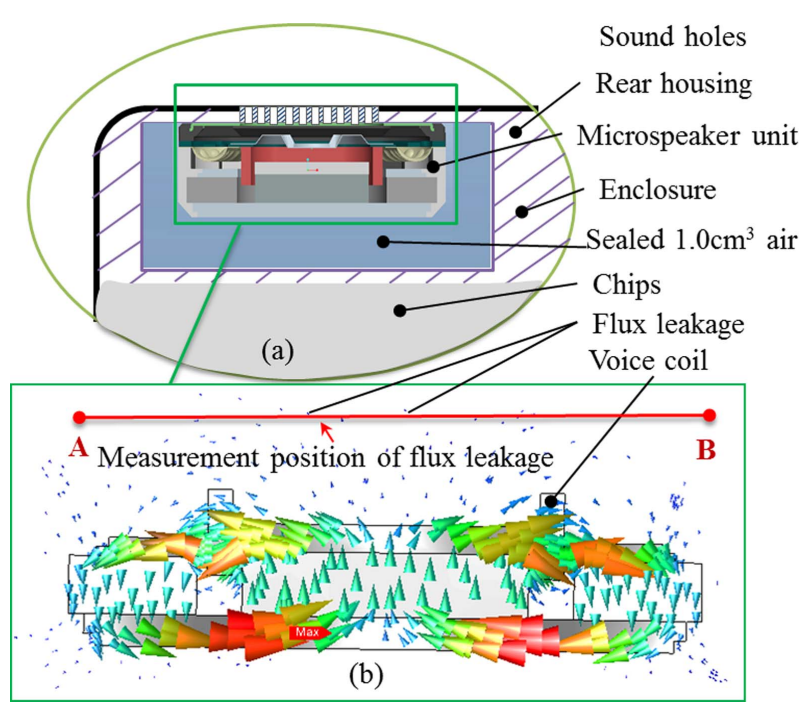

Fig. 1. (Color online) (a) Partial section of the arrangement of a microspeaker in a mobile phone, and (b) A section of flux vectors on the magnetic circuit in a microspeaker. 


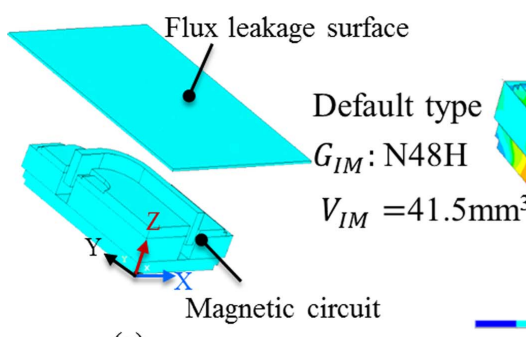

(a)

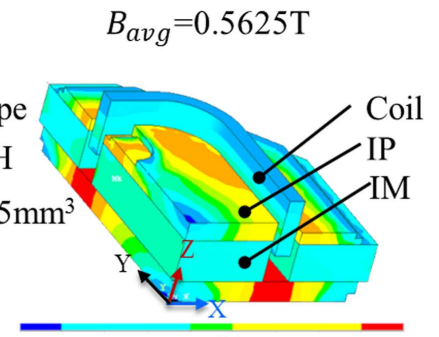

(b)

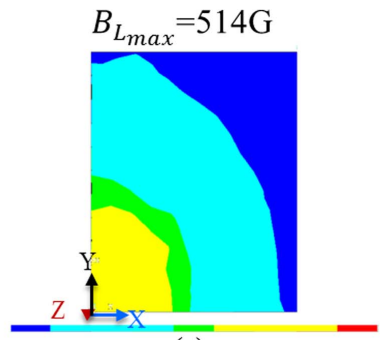

(c)

Fig. 2. (Color online) Default Type of (a) Electromagnetic analysis modeling, (b) Flux density distribution and (c) Flux leakage distribution.

Table 1. Parameters of default type.

\begin{tabular}{lclc}
\hline \multicolumn{1}{c}{ Parameter } & Value & \multicolumn{1}{c}{ Parameter } & Value \\
\hline Coil diameter $(\mathrm{mm})$ & 0.093 & Total back volume $\left(\mathrm{cm}^{3}\right)$ & 1.0 \\
Coil length $(\mathrm{mm})$ & 1463 & Effective DP area $\left(\mathrm{mm}^{2}\right)$ & 102.4 \\
Dynamic mass $(\mathrm{mg})$ & 54.72 & Speaker unit stiffness $(\mathrm{N} / \mathrm{m})$ & 350 \\
Air density $\left(\mathrm{kg} / \mathrm{m}^{3}\right)$ & 1.2 & Equivalent stiffness $(\mathrm{N} / \mathrm{m})$ & 1455 \\
Sound velocity $(\mathrm{m} / \mathrm{s})$ & 340 & Total stiffness $(\mathrm{N} / \mathrm{m})$ & 1805 \\
Input power $(\mathrm{W})$ & 0.64 & $\mathrm{f}_{0}(\mathrm{~Hz})$ & 914 \\
\hline
\end{tabular}

four magnetic circuit designs are proposed to reduce the flux leakage:

Type A: An IM with a smaller volume.

Type B: An IM with a lower grade magnet level.

Type C: A hole punched in the IM.

Type D: A hole punched in both the IM and IP.

To make the four proposed designs comparable, maintain performance within an imperceptible level of the human auditory system [4], and satisfy the IM dimensions and machining allowances, the following specifications were established. (1) The outer dimension of the IM in Type A was $0.13 \mathrm{~mm}$ smaller than that of the default design. (2) An N33H-grade magnet was applied to the IM in Type B because of the grade limit of Nd-Fe-B sintered permanent magnets. (3) Consider the additional price of additional manufacturing process in mass production and the hardness of IM with a punched hole, a circular hole is executed in Type C and Type D. (4) In order to keep the same IM volume as that of Type A, Ø2.70 mm circular holes were selected for Type $\mathrm{C}$ and Type $\mathrm{D}$, as shown in Figs. 3(a1), (b1), (c1), and (d1). Electromagnetic analyses were conducted by using the finite element method (FEM) to obtain the magnetic flux density and the flux leakage distributions. An optimization analysis of the adopted method in the four proposed types was implemented by using the response surface method (RSM) [5]. Five samples were manufactured on the basis of the simulations. The sound pressure level (SPL) is used to characterize the acoustic performance [6]. A Kanetec Gauss meter and $\mathrm{B} \& \mathrm{~K}$ equipment were employed for the flux leakage and

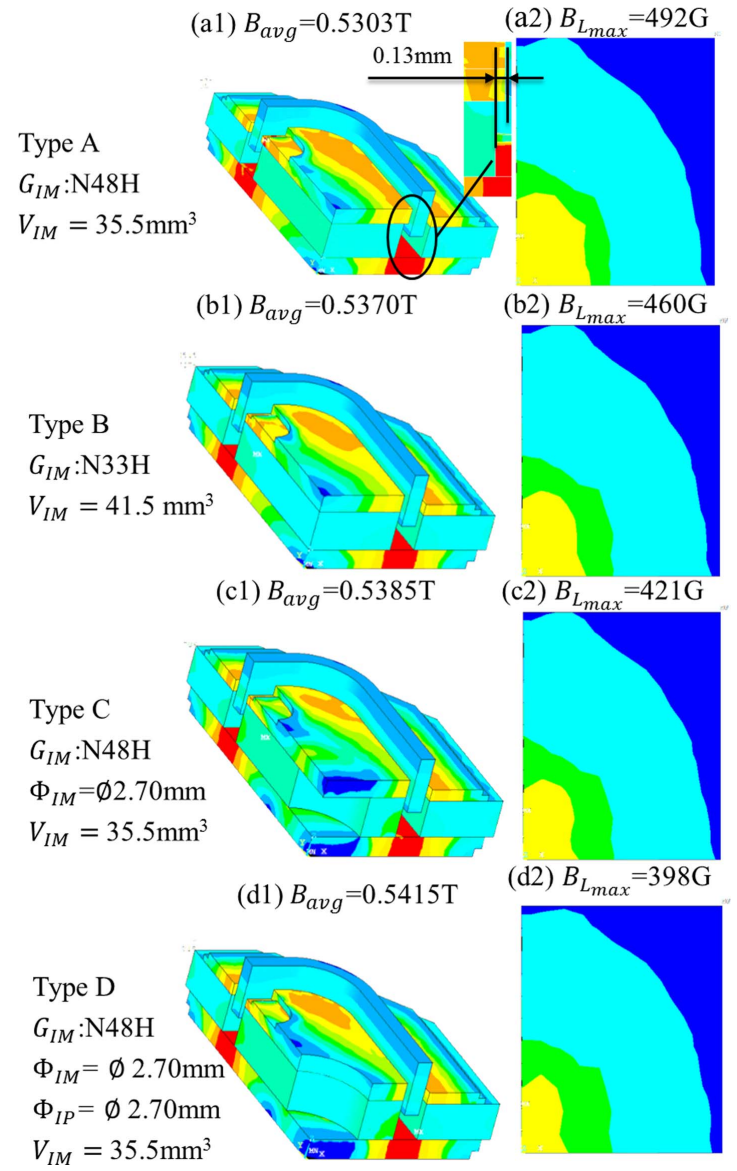

Fig. 3. (Color online) Flux density distributions on the lefthand side and flux leakage distributions on the right-hand side of proposed Type A, B, C, and D.

SPL experiments. The SPL performance and the maximum reduction of flux leakage were confirmed experimentally.

\section{Analysis Methods and Discussion}

\subsection{Electromagnetic Analysis}

To analyze the electromagnetic system, a one-quarter three-dimensional (3D) modeling was constructed on the symmetric magnetic circuit's vertical and horizontal axes, 
as shown in Fig. 2(a). The Fig. 2(b) and (c) are the electromagnetic analysis contour results of magnetic circuit and front leakage surface in Fig. 2(a) by using the FEM with commercial ANSYS program. To make it more easily to understand, the top view of Fig. 2(a) is displayed in Fig. 2(c). As it's shown in Fig. 3, four design methods as previous mentioned were proposed on the IM of the magnetic circuit because the center area has higher flux leakage than other areas of the flux leakage distribution, and has a lower flux density on the magnetic circuit in the flux density distribution of the default design, as shown in Figs. 2(b) and (c), respectively. In this paper, all of the simulations of $B_{\text {avg }}$ have the same scaling and view angle as the Fig. 2(b), and all of the simulations of $B_{\mathrm{Lmax}}$ have the same scaling and view angle as the Fig. 2(c). Particularly, the Type D design was punched with the same diameter circular hole in the IP because the flux density on the center area of the IP in Type $\mathrm{C}$ was much smaller than that of other areas. Compared with the default type, even though the flux density was decreased dramatically, the flux leakage reduction of Type A was not obvious, as shown in Fig. 3(a2). The flux leakage reduction of Type B, as shown in Fig. 3(b2), was evident with an expected flux density sacrifice. As shown in Fig. $3(\mathrm{c} 2)$ and (d2), the Type $\mathrm{C}$ design reduced much more flux leakage than that of Type B with a similar flux density distribution. Type $\mathrm{D}$ was improved with a higher flux density and a lower flux leakage, based on Type C. In addition, the decreased $V_{\mathrm{IM}}$ in Type $\mathrm{A}$ and Type $\mathrm{D}$ filled with air increased the sealed $1.0 \mathrm{~cm}^{3}$ back volume of the speaker module, and slightly improved the acoustic performance of both types.

\subsection{Vibration and Acoustic Analysis}

The acceleration of the diaphragm's surface was obtained through mechanical analysis using Eq. (1), where $[M]$, $[C],[K],\left\{f_{\text {coil }}\right\}$ denote the mass matrix, damping matrix, stiffness matrix, and force vector generated in the coil, respectively. A Lorentz force is generated when a current passes through the voice coil, and can be expressed by Eq. (2), where $F_{\text {coil }}, I, l, B_{\text {avg }}$ denotes the magnetic excitation forces acting on the voice coil, the coil current, the total coil length, and average radial magnetic flux density in the air gap, respectively.

$$
\begin{aligned}
& {[M]\{\ddot{z}\}+[C]\{\dot{z}\}+[K]\{z\}=\left\{f_{\text {coil }}\right\}} \\
& F_{\text {coil }}=\oint I d l \times B_{\text {avg }}
\end{aligned}
$$

The SPL can be obtained by using Eq. (3), where $p_{r m s}$ and $p_{\text {ref }}$ denote the pressure generated by the diaphragm and the reference pressure, $20 \mu \mathrm{Pa}$, respectively. $p_{r m s}$ can be obtained by using Eq. (4), where $\rho_{0}, r, d$ and $a_{0}$ denote the air density, effective radius of the diaphragm, distance of measurement, and acceleration caused by magnetic force, respectively.

$$
\begin{aligned}
& S P L=20 \log \frac{p_{r m s}}{p_{r e f}} \\
& \left|p_{r m s}\right|=\left(\frac{\rho_{0} r^{2}}{2 d}\right)\left|a_{0}\right|
\end{aligned}
$$

According to the simulations and analyses, the performance of the microspeaker in the Type A design was degraded with a $4.3 \%$ reduction in $B_{\mathrm{L}_{\max }}$ and a 0.54 $\mathrm{dB}$, which is discernible to the human ear, decrease at the primary acoustic resonance compared with the default type. Based on the simulations, the proposed Type D design reduced the flux leakage the most while sacrificing the least flux density among the four proposed types.

\section{Optimization and Discussion}

Optimization of the proposed Type D design was implemented by using the face-centered central composite design of RSM with four steps. First, the diameters of the circular hole punched in the IM and IP with three levels were varied in a selected range and were set as $\xi_{\mathrm{A}}, \xi_{\mathrm{B}}$ of natural variables and $x_{\mathrm{A}}, x_{\mathrm{B}}$ of coded variables as in Table 2. Second, $B_{\text {avg }}$ and $B_{\mathrm{L}_{\max }}$ were chosen as the objective functions in order to investigate acoustic performance at the primary resonance frequency and the maximum reduction in flux leakage. Third, to acquire a more precise optimized value, ranges from $2.8 \mathrm{~mm}$ to $3.2 \mathrm{~mm}$ were selected for the both natural variables based on interaction effect analyses. Finally, the performances of each set shown in Table 2 were estimated by using the FEM, similar to the default design and the four proposed designs. The response surfaces of $B_{\text {avg }}$ and $B_{\mathrm{L}_{\max }}$ with coded variables were fitted by Minitab 16, as shown in Figs. 4(a) and (b). The final model in terms of both the coded variable and the natural variable levels can be computed out by the software package. The polynomial approximation models were determined for the functional relationships between the performance characteristics and the design variables whose fitted coded second-order model is expressed as follows:

$$
\begin{aligned}
& B_{\text {avg }}\left(x_{\mathrm{A}}, x_{\mathrm{B}}\right)=0.5360-0.00465 x_{\mathrm{A}}+0.000517 x_{\mathrm{B}} \\
& \quad-0.00065 x_{\mathrm{A}}^{2}-0.00025 x_{\mathrm{B}}^{2}+0.00055 x_{\mathrm{A}} x_{\mathrm{B}} \\
& B_{\mathrm{L}_{\max }}\left(x_{\mathrm{A}}, x_{\mathrm{B}}\right)=372.100-12.617 x_{\mathrm{A}}-3.617 x_{\mathrm{B}} \\
& \quad+2.050 x_{\mathrm{A}}^{2}-12.750 x_{\mathrm{B}}^{2}+9.900 x_{\mathrm{A}} x_{\mathrm{B}}
\end{aligned}
$$

while considering the constrained objective functions, the 
Table 2. DOE about the punched hole diameters.

\begin{tabular}{|c|c|c|c|c|c|c|}
\hline \multirow{2}{*}{ No. } & \multicolumn{2}{|c|}{ Natural variables } & \multicolumn{2}{|c|}{ Coded variables } & \multicolumn{2}{|c|}{ Responses } \\
\hline & $\xi_{\mathrm{A}}$ & $\xi_{\mathrm{B}}$ & $x_{\mathrm{A}}$ & $x_{\mathrm{B}}$ & $B_{\text {avg }}(\mathrm{T})$ & $B_{\mathrm{L} \max }(\mathrm{G})$ \\
\hline 1 & 2.8 & 2.8 & -1 & -1 & 0.5398 & 386.5 \\
\hline 2 & 3.2 & 2.8 & 1 & -1 & 0.5293 & 339.3 \\
\hline 3 & 2.8 & 3.2 & -1 & 1 & 0.5398 & 363.7 \\
\hline 4 & 3.2 & 3.2 & 1 & 1 & 0.5315 & 356.1 \\
\hline 5 & 2.8 & 3.0 & -1 & 0 & 0.5399 & 384.6 \\
\hline 6 & 3.2 & 3.0 & 1 & 0 & 0.5308 & 363.7 \\
\hline 7 & 3.0 & 2.8 & 0 & -1 & 0.5353 & 367.2 \\
\hline 8 & 3.0 & 3.2 & 0 & 1 & 0.5362 & 351.5 \\
\hline 9 & 3.0 & 3.0 & 0 & 0 & 0.5360 & 372.1 \\
\hline
\end{tabular}

optimal values of $\Phi_{\mathrm{IM}}$ and $\Phi_{\mathrm{IP}}$ can be determined by the Eq. (5), which are 0.75 and -0.90 in coded levels, and $3.15 \mathrm{~mm}$ and $2.82 \mathrm{~mm}$ converts in natural levels. The objective functions $B_{\text {avg }}$ and $B_{\mathrm{L} \max }$ are predicted to be $0.5314 \mathrm{~T}$ and $367 \mathrm{G}$, respectively, based on the simulation results, as shown in Fig. 5. The $B_{\mathrm{L}_{\max }}$ of the optimized type is decreased by $28.6 \%$, which is $6.1 \%$ more than the proposed Type D. Even though the $5.5 \% B_{\text {avg }}$ reduction of the optimized design is larger than that of proposed Type $\mathrm{D}$, it still can maintain the acoustic
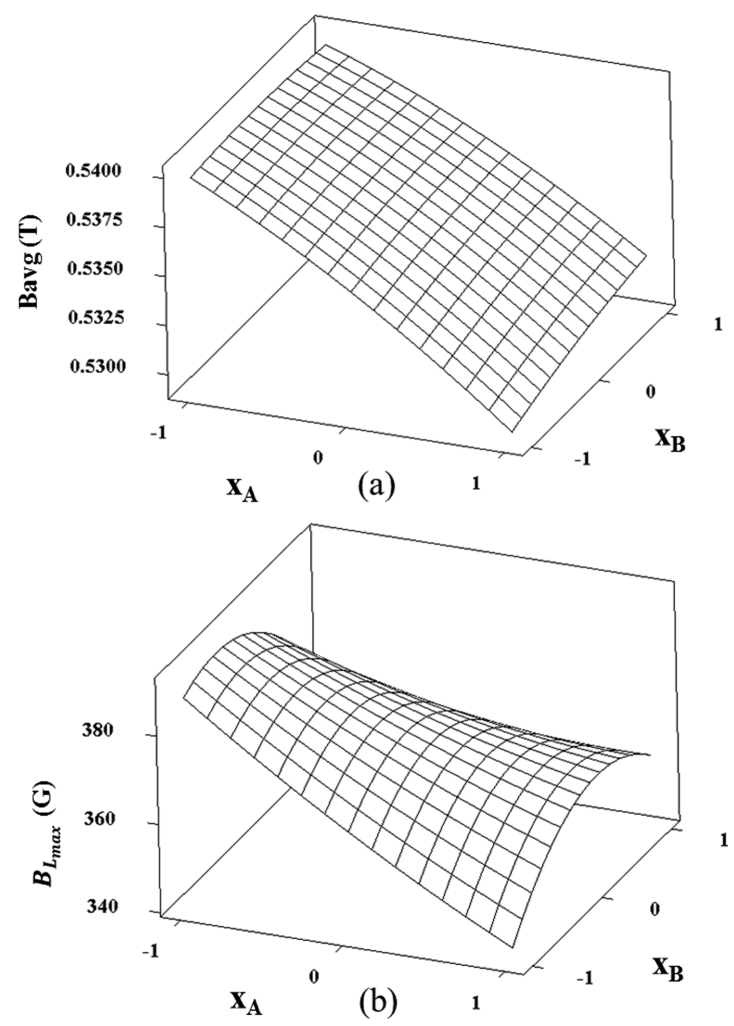

Fig. 4. Response surface plot of (a) Average of radial flux density passing through coil and (b) Maximum flux leakage on the surface above the speaker unit for $1 \mathrm{~mm}$.

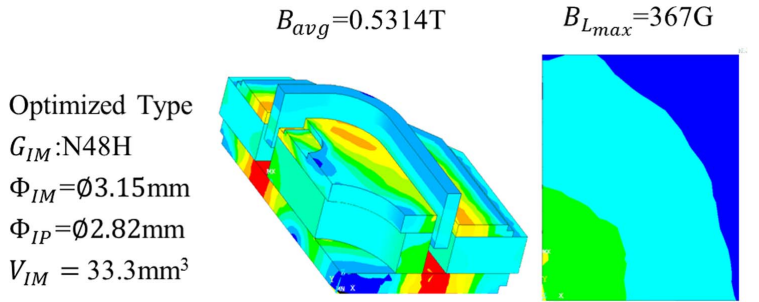

Fig. 5. (Color online) Optimized type of (a) Flux density distribution and (b) Flux leakage distribution.

performance within an imperceptible auditory gap because the SPL at the primary resonance frequency is only reduced by $0.45 \mathrm{~dB}$ as the simulated.

\section{Experimental Results and Discussion}

Samples were built on the basis of the simulation results. Figure 6 shows a photograph of the default design, and five types of speaker units and their components. Type A was excluded because of its undesirable slight reduction of flux leakage and unacceptable decrease in acoustic performance in simulations. Figure 7(a) shows the setup of the Kanetec Gauss meter (TM-501) for measuring the maximum flux leakage on the plastic baffle $1 \mathrm{~mm}$ above the speaker unit. Figure 7(b) shows the setup of the SPL experiment measurement using a B\&K pulse system (3560C) that receives the input signals in a microphone positioned $10 \mathrm{~cm}$ away. As it's shown in Table 3, the simulated results are in good agreement with the experimental results. The optimized design reduced the value of $B_{\mathrm{L}_{\max }}$ by $28.6 \%$ with only a $0.0311 \mathrm{~T}$ in $B_{\text {avg }}$ and a 0.45 $\mathrm{dB}$ SPL decrease at the primary resonance. This is within the indiscernible gap of the human ear. Figure 8 shows the SPL graph of the simulated and experimental results

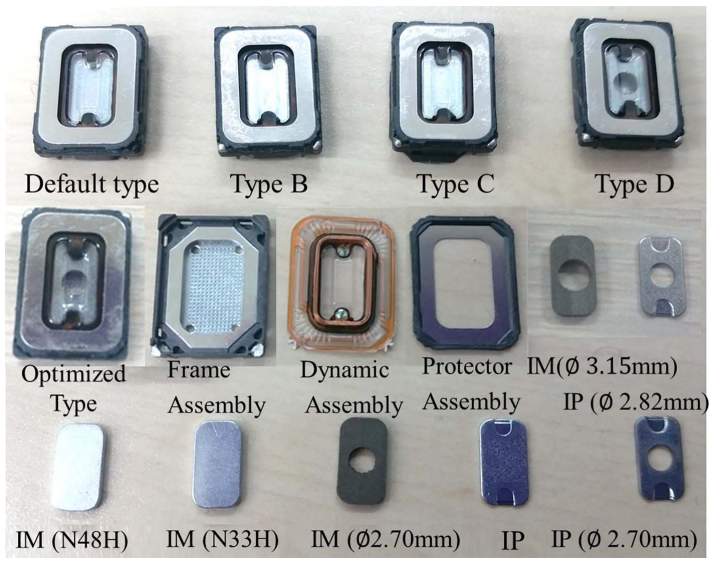

Fig. 6. (Color online) Samples of four types: default type, proposed Type B, C and D. 

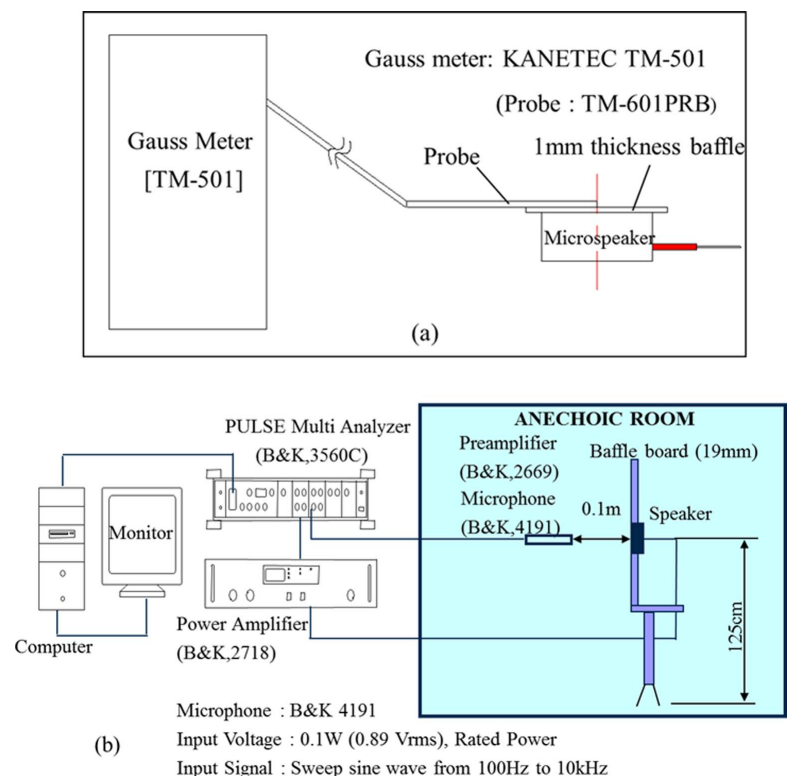

Fig. 7. (Color online) Experimental setups for testing (a) Maximum flux leakage and (b) SPL.

for the default design and optimized design, and shows that the experimental results are in good agreement with those of the one degree-of-freedom analysis from $100 \mathrm{~Hz}$ to $5 \mathrm{kHz}$. Figure 9 shows the experimental SPL graph of the five designs, and indicates that the acoustic performance of proposed Type $\mathrm{D}$ design achieves the design goal much more effectively than the other types. In addition, both of the differences in the SPL curves between the default design and the Type $\mathrm{D}$ or optimized design at any frequency from $100 \mathrm{~Hz}$ to $20 \mathrm{kHz}$ is within an imper-

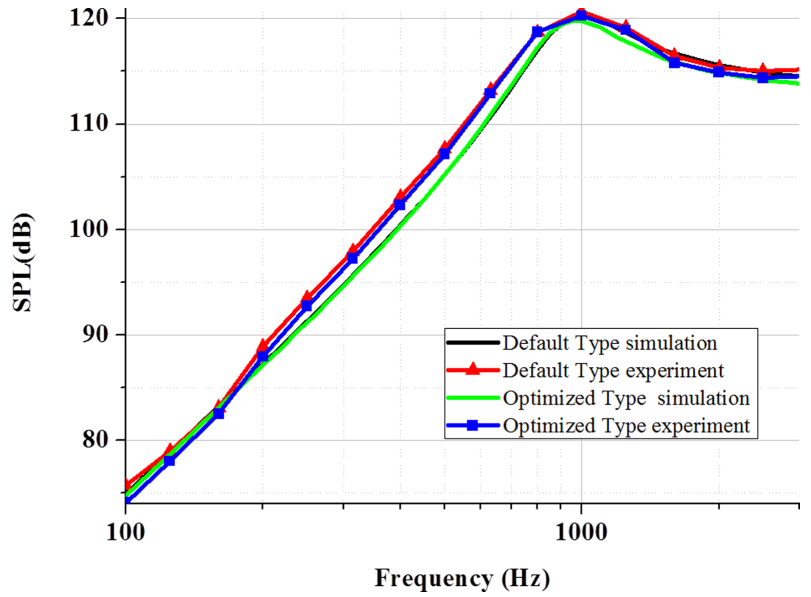

Fig. 8. (Color online) Simulated and experimental results comparison of default type and optimized type.

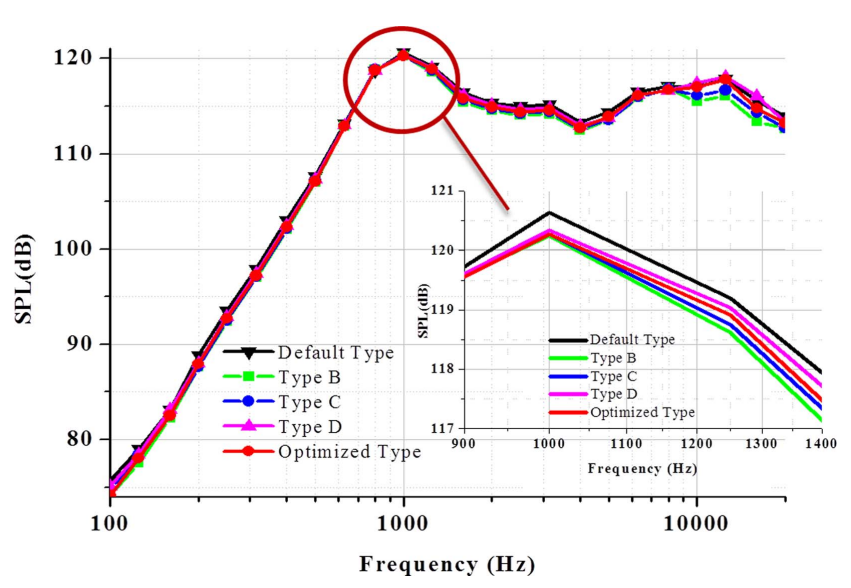

Fig. 9. (Color online) Comparison of experimental SPL results.

Table 3. Simulated and experimental results.

\begin{tabular}{|c|c|c|c|c|c|c|c|}
\hline & Type & Default type & Type A & Type B & Type C & Type D & Optimized type \\
\hline & $G_{\mathrm{IM}}$ & $\mathrm{N} 48 \mathrm{H}$ & $\mathrm{N} 48 \mathrm{H}$ & $\mathrm{N} 33 \mathrm{H}$ & $\mathrm{N} 48 \mathrm{H}$ & $\mathrm{N} 48 \mathrm{H}$ & $\mathrm{N} 48 \mathrm{H}$ \\
\hline & $\operatorname{Br}(\mathrm{T})$ & 1.36 & 1.36 & 1.14 & 1.36 & 1.36 & 1.36 \\
\hline & $\mathrm{Hc}(\mathrm{kA} / \mathrm{m})$ & 890 & 890 & 844 & 890 & 890 & 890 \\
\hline & $\Phi_{\mathrm{IM}}(\mathrm{mm})$ & 0 & 0 & 0 & 2.70 & 2.70 & 3.15 \\
\hline & $\Phi_{\mathrm{IP}}(\mathrm{mm})$ & 0 & 0 & 0 & 0 & 2.70 & 2.82 \\
\hline & $V_{\mathrm{IM}}\left(\mathrm{mm}^{3}\right)$ & 41.5 & 35.5 & 41.5 & 35.5 & 35.5 & 33.3 \\
\hline & $B_{\text {avg }}(\mathrm{T})$ & 0.5625 & 0.5303 & 0.5370 & 0.5385 & 0.5415 & 0.5314 \\
\hline & $B_{\text {avg }}$ Reduction & & $5.7 \%$ & $4.5 \%$ & $4.3 \%$ & $3.7 \%$ & $5.5 \%$ \\
\hline \multirow{4}{*}{ Simulated } & $B_{\mathrm{L}_{\max }}(\mathrm{G})$ & 514 & 492 & 460 & 421 & 398 & 367 \\
\hline & $B_{\mathrm{L}_{\max }}$ Reduction & & $4.3 \%$ & $10.4 \%$ & $18.1 \%$ & $22.5 \%$ & $28.6 \%$ \\
\hline & $f_{0}(\mathrm{~Hz})$ & 914 & 912 & 914 & 914 & 912 & 911 \\
\hline & $\mathrm{SPL}$ at $f_{0}(\mathrm{~dB})$ & 120.26 & 119.77 & 119.90 & 119.92 & 119.98 & 119.81 \\
\hline \multirow{3}{*}{ Experimental } & $B_{\mathrm{L}_{\max }}$ Reduction & & & $10.2 \%$ & $17.8 \%$ & $23.6 \%$ & $29.7 \%$ \\
\hline & $f_{0}(\mathrm{~Hz})$ & 909 & & 910 & 911 & 907 & 907 \\
\hline & SPL at $f_{0}(\mathrm{~dB})$ & 120.64 & & 120.25 & 120.28 & 120.34 & 120.27 \\
\hline
\end{tabular}


ceptible auditory gap. The punched hole on the magnetic circuit increased the back volume by $6 \mathrm{~mm}^{3}$, which can improve the acoustic performance slightly [6].

\section{Conclusion}

Compared with using a smaller magnet volume in the Type A design or a lower grade magnet in the Type B design, the proposed method with a circular hole punched in both the IM and IP is more effective in reducing flux leakage while sacrificing less flux density. These results are also better than those of the case with a circular hole punched only in the IM. The optimized design, based on the proposed Type D design and using RSM, reduces the maximum flux density to a much greater degree than the Type $\mathrm{D}$ design alone. The optimized design reduces the maximum flux leakage by $28.6 \%$ while only decreasing the average radial flux density by $5.5 \%$ and the primary resonant SPL by $0.45 \mathrm{~dB}$. Moreover, the proposed Type D method can improve the acoustic performance slightly with an increased back volume, and can be carried out easily, quickly, and economically without much extra structure design. The electromagnetic analyses were performed by using the FEM and the simulated results were validated experimentally.

\section{Acknowledgments}

This work was supported by a 2-Year Research Grant of Pusan National University.

\section{Nomenclature}

IM : Inner magnet

IP : Inner plate

$G_{\mathrm{IM}} \quad$ : Grade of Nd-Fe-B Sintered permanent inner magnet

$V_{\mathrm{IM}} \quad$ : Volume of inner magnet

$\Phi_{\text {IM }} \quad$ : Diameter of circular hole punched on IM

$\Phi_{\mathrm{IP}} \quad$ : Diameter of circular hole punched on IP

$\mathrm{f}_{0} \quad$ : Primary resonance frequency

$B_{\text {avg }}$ : The average radial flux density in the air gap passing through the voice coil

$B_{\mathrm{L}_{\max }}$ : The maximum value on the flux leakage distribution surface

\section{References}

[1] Ali Emadi, Advanced Electric Drive Vehicles, CRC Press, USA (2014).

[2] Dan-Ping Xu, Peng Sun, Joong-Hak Kwon, and SangMoon Hwang, J. Appl. Phys. 115, 17A339 (2014).

[3] Sun, Peng, Dan-Ping $\mathrm{Xu}$, and Sang-Moon Hwang, J. Mech. Sci. Technol. 28, 1623 (2014).

[4] David V. Chadderton, Building Services Engineering, $4^{\text {th }}$ Edition, Taylor \& Francis e-Library (2004).

[5] Douglas C. Mountgomery, Design and Analysis of Experiments, $6^{\text {th }}$ ed., John Wiley $\&$ Sons, Inc. (2005).

[6] Kinsler, Lawrence E., Austin R. Frey, Alan B. Coppens, and James V. Sanders, Fundamentals of Acoustics, 4th ed., New York, Wiley (1999). 